\title{
Geographisches Register
}

Aachen 9

Aalen $147,481 \mathrm{ff} ., 486 \mathrm{f} ., 496,500 \mathrm{ff} ., 505$, $515,518,547,593$

Aichach $147,164,167,239,286,291,294$

Alsfeld $327,341,343,345,347 \mathrm{f}$., $397 \mathrm{f}$., $400 \mathrm{ff}$., 443, 447

Altmühl 146

Altötting 164, 167, 269,286, 292, 294, 308, 314

Alzenau i. UFr. 163, 165, 284, 290, 293

Amberg 164, 166, 196, 285, 288, 291, 294, 313

Ammersee 308

Ansbach 145, 150, 156, 163, 165, 168, 212 , 217, 236, 256, 284f., 290, 293, 299, 308, 313

Apolda 10

Arolsen 343f., 443

Aschaffenburg 146, 163, 165, 284, 288, 290 , $293,308,312$

Assmannshausen 369

Aubing 164

Augsburg 145, 147, 150, 156, 163f., 168f., $174,196,199,201,217,236,241,243 f ., 249$, 256, 269, 272, 284, 286ff., 292, 294 f., 315

Babelsberg $677 \mathrm{f}$.

Backnang 481 ff., 486f., 495, 500, 502f., 505, $515,518,535,538,547,592$

Bad Aibling $162,164,167,286,292,294,308$, 314

Bad Cannstatt 578

Bad Ems 345

Bad Hersfeld 343, 345, 347f., 397f., 400 ff., $444 f ., 447$

Bad Homburg v. d. Höhe 52, 325, 344, 346f., 398, 401 f., 414, 443, 447, 488, 510, 537

Bad Kissingen 108f., 163, 165, 284, 289, 293, 312

Bad Mergentheim 467, 481 ff., 486f., 496, $500,502,505,515,518,535,538,547,569$, $577,593 \mathrm{f}$.

Bad Nauheim $63,70,76,93,97,107,112$, 114, 116, 119, $374 \mathrm{f} ., 379$

Bad Neustadt a.d. Saale $163,165,284,293$, 313

Bad Orb 325, 344, 443, 649

Bad Reichenhall 154

Bad Schwalbach $325,344,346,397 \mathrm{f} ., 402 \mathrm{f}$., $443 f$.

Bad Tölz 10, 147, 164, 167, 196, 199, 286, 292, 294, 308, 314

Bad Wildungen 344,346

Bad Wimpfen 568, 59

Bad Wörishofen 308
Baden 457f., 460, 475, 496, 509f., 536, 541, $576,585,588$

Baden-Baden 127

Bamberg $145,150,163,168,196,202,246 \mathrm{f}$. $249,269,285,288,290,293,308,313,572$

Barbizon 675

Bayem 10, 28, 44, 75, 86, 143-315, 368f. $376,384,457,489,536,569,573,587$

- Mittel- und Oberfranken $73,147,156$, $163,165,170,172,236,283$ ff., $289 f$., 295 , $299,311,313,587$

- Niederbayern und Oberpfalz 73, 147, 156, 164, 166, 170, 172, 236, 283 ff., 288 ff., 293, 295, 311

- Oberbayern 73, 146f., 157, 162, 164, 167 . $170,172,236,283$ f., 286, 289, 291, 294 f., 311,314

- Schwaben $73,146,148,156,164,168,170$. $172,204,236,283$ f., 286, 289, 292, 294 f., 311,314

- Unterfranken $73,147,157,162 \mathrm{f}, 165,170$, $172,207,236,283$ f., 289, 293, 295, 306, 311

Bayreuth $146,163,166,202,246,285,288$, $290,293,308,313$

Bebra 424

Beerfelden 325, 344, 443

Beilngries 164, 166, 286, 291, 294

Bensheim 343, 345, 347f., 386, 443

Berchtesgaden 146, 164, 167, 286, 288, 292. 294, 314

Bergstraße $343,345,347 \mathrm{f} ., 397-402,447,596$

Bergzabem 575

Berlin 4,10, 12, 18, 24, 26, 28, 34f., 38, 41, $43 \mathrm{f}$., 53, 56, 58, 73, 75f., 83, 87f., $92 \mathrm{f} ., 96 \mathrm{f} ., 99,105$, $107,112-116,120,124,127,131,135$ ff., 245 , $251,253,358,376,409,606,671-738$

- Britischer Sektor 673,676,681

- Französischer Sektor 681

- Sowjetischer Sektor 673, 681, 697, 711, $714,716,720,736,738$

- Dahlem 678, 682, 688

- Díppel 728

- Friedenau $701 \mathrm{f} ., 714$

- Gatow 678

- Grunewald 678

- Karlshorst 678

- Köpenick 674

- Kreuzberg 680, 688, $691 \mathrm{ff} ., 701 \mathrm{ff}$.

- Lichterfelde 679f., 684, 686, 710,713

- Neukölln 680, 688,691 ff., 701 ff., 714

- Schöneberg 680, 686, 688, 691 ff., 701 ff., 710f., 714, 738 
- Spandau 689f.. 712, 714,736

- Steglitz 680, 687 f., 691 ff., 701 ff., 714, 730, 736

- Tempelhof $677 \mathrm{f} ., 680,688,691 \mathrm{ff} ., 701 \mathrm{ff}$., 714

- Tiergarten 737

- Wilmersdorf 736

- Zehlendorf $680,688,691 \mathrm{ff} ., 701 \mathrm{ff} ., 714$, 728, 736

Biedenkopf $325,344,346 \mathrm{f} ., 396,400 \mathrm{ff} ., 443$, 447

Bielefeld 52, 56, 62, 124, 341, 488, 676

Bietigheim 518

Blaubeuren 518,593

Böblingen $481 \mathrm{ff}$., $486 \mathrm{f}$., 495, 499, $502 \mathrm{f}$., 505 . $515,518,547,578,592$

Bogen 164, 167, 286, 291, 294, 313

Bonn 733

Brake 605, 609f., 614f., 617, 635, 655

Brandenburg 4

Braunau a. Inn 146

Braunschweig 622

Bremen $10,44,52,73,75,87,122,127,136$, 139. $275,369,533,597-670,679$

Bremerhaven 135, 601-612,614-617,619, $621-624,626 \mathrm{f} ., 630,632-635,642,645,650$, $652,655 f ., 662-666,668,670$

Bretten 594

Bristol Channel 612

Britische Besatzungszone $131,138,420,424$, $458,569,600-604,606 \mathrm{ff} ., 610,615-624,629$, $673,711 \mathrm{f}$.

Bruchsal $481 \mathrm{ff} ., 486 \mathrm{f} ., 495,500,502,505$, S16f., 535, 538, 546, 573, 575, 595

Brückenau 163, 165, 284, 289, 293, 312

Buchen (Odenwald) 481 ff., $486 f ., 495$, 500ff., 505, 515, 517, 547, 569, 595

Bildingen 343, 345, 347f., 397-402, 443

Burghausen 164

Burglengenfeld 164, 166, 285, 291, 294, 313

Bushy Park 107

Butzbach 428

Camberg 388

Camp Custer 457

Carlisle Barracks, Pa. 553

Cham 164, 166, 285, 291, 294, 313

Charlottesville, Va. 5, 457

Chartres 152

Châtillon sur Seine 457

Celle 619

Coburg 146, 163, 166, 249, 285, 288, 290, 293, 308,á 313

Crailsheim 481 ff., 486 f., 496, 500, 502. 505, $515,518,535,538,547,569,593$
Dachau 146, 164, 167, 286, 291. 294, 308. 314

Darmstadt (siehe auch Hessen/RB Darmstadt) $109,324,340-343,345,347 f ., 358$. 377-380, 386, 388, 391 f., 397-402, 405, 414. $424,428 f$. 439,442 f., $445,447,450,666$

Deggendorf $164,167,285,291,294,313$

Delmenhorst 607f., 623

Dieburg 343, 345, 347f., 397-402, 443, 447

Dill-Kreis $325,344,346 f ., 396,398 f ., 401 \mathrm{f}$., 447

Dillenburg 344, 346f., 396, 398, $401 \mathrm{f} ., 443$

Dillingen a.d. Donau 145, 147, 164, 168, 287. 292, 295, 315

Dingolfing 164, 167. 286, 291, 294

Dinkelsbühl $163,165,285,290,293,313$

Donau $145 \mathrm{ff}$.

Donauwörth $145,147,164,168,287,292$. 294,315

Dorfen $146 \mathrm{f}$.

Düsseldorf 127

Durlach $512 \mathrm{f}$.

Eberbach 517

Ebermannstadt $163,166,285,290,293,313$

Ebern 163, 165, 284. 290.293, 313

Ebersberg 164, 167, 286, 292

Edingen 517, 557f., 595

Eger 148

Eggenfelden 164, 167, 285, 291, 294, 313

Ehingen (Donau) 145

Eichstätt $163,165,285,290,293,313$

Eisenach 10

Elbe 677

Elsfleth 605

Enns 146

Enschede 608

Erbach (Odenwald) 327, 343 ff., 347f., 397402,443

Erding 164, 167, 286, 291, 294

Erfurt 10

Erlangen $147,151,156,163,166,249,285$, $290,293,306,313$

Esch a.d. Alzette 321, 341

Eschenbach i.d. OPf. $164,166,285,291,294$

Eschwege 343, 345, 347, 397f., $401 \mathrm{f} ., 443,447$

Essen 71, 345

Esslingen 467, $481 \mathrm{ff}, \mathbf{4 8 6 f}$., 495, 499, 502. $505,515,518,535,538,547,556,560,592$

Etain 320

Ettlingen 517,594

Fellbach 518, 592

Feuchtwangen $163,165,285,290,293,313$

Flensburg 15

Flörsheim a. Main 325, 344, 443

Forchheim 163, 166, 285. 290, 293 
Franken siehe Bayem/Mittel-, Ober- und Unterfranken

Frankenberg $325,343,345,347,397 f ., 400$ ff. 443, 447

Frankfurt a. Main 20f.. 26, 28, 35, 38, 41, 43. 52f., $56 \mathrm{ff} ., 60,71,78,82,88,91,99 \mathrm{f} ., 105$. 107. 109, 112-116, 124f.. 151, 251 f., 322. 325. 330, 336, 340ff., 346f., 350, 361, 363 , $368,375-380,386,388,391 \mathrm{f} ., 396,398-403$, $405,407,409 \mathrm{ff}, 414,424,435 \mathrm{f} ., 442,445$, $447,450,561,687,723$

- Höchst $18,26,28,62,71,126,678$

Frankreich $600 f$.

Französische Besatzungszone 15, 147, 420, $424,458-462,475,521,536,562,564,569$. $575 \mathrm{f}, 588,711 \mathrm{f}$.

Freiburg i. Breisgau 564, 576

Freilassing 308

Freising 164, 167, 253, 286, 291, 294

Freyung 164, 286, 291

Friedberg (Bayem) 147, 164, 168, 187, 292, 294, 315

Friedberg (Hessen) 322, 343, 345, 347f., 392, $397-402,444,447$

Fritzlar 343, 345, 397f., 400 ff., 444, 447

Fritzlar-Homberg $343,345 \mathrm{ff} ., 397 \mathrm{f}$., $400 \mathrm{ff}$.

Fürstenfeldbruck 164, 167, 286, 291, 294, 308,314

Fürstenhagen 34

Firth $163,165,196,279,285,290,293,313$

Füssen $164,168,287,292,295,315$

Fulda 343,345 ff., 377-380, 386, 397f., $400 \mathrm{ff}$., 444,447

Garmisch-Partenkirchen $164,167,196,286$, 291, 294, 314

Geislingen a.d. Steige $\$ 92$

Gelnhausen 325, 344, 346f., 396, 398f., $401 \mathrm{f}$., 444

Gemünden a. Main $163,165,284,290,293$

Gendorf 272, 275, 279

Georgenborm 374

Gera 10

Gerolzhofen $163,165,284,289,293$

Gießen $341,343,345,347,377,379 f ., 386$, $388,397 \mathrm{f} ., 400 \mathrm{ff} ., 414,424,444,447 \mathrm{f}$.

Göppingen 468, $481 \mathrm{ff} ., 486 \mathrm{f}$., 496, 500-503. $505,515,518,535,538,547,559,592 \mathrm{f}$.

Gotha 10

Grafenau $147,164,167,285,291,294,313$

Griesbach i. Rottal 164, 167,286,291,294,313

Griesheim 386

Grohn 612,616

GroB-Gerau 327, 343, 345, 347f., 397-402, 444, 447

Grünberg 343,444
Günzburg $147,164,168,287,292,295,315$

Gütersloh 675

Gunzenhausen $163,165,285,290,293$

Hadamar 325. 344

Halle a.d. Saale $675,677 \mathrm{f}$.

Hamburg 52, 127, 601, 619f., 622f., 626

Harnmelburg 163, 165, 284, 289, 293, 313

Hanau $322,325,344,346 f ., 396,398-402$. $414,444,447$

Hannover $127,610,617-620,622,642$

Haßfurt $163,165,284,289,293,313$

Heidelberg 10, 91, 147, 322, $467 \mathrm{ff} ., 481 \mathrm{ff}$., 486f., 495, 500-503, 505, 509, 511, 515, 517, $524,532,534-538,542 f$., 546, 552, 555, 560 , $563 f$., 566, 573, 575, $577 f$., 590, 595

Heidenheim a.d. Brenz 147, 481 ff., 486 f., 496, 500ff., 505, 515, 518, 535, 538, 547, 593

Heilbronn 467 ff., 481 ff., $486 f$., $496,500 f$., $503,505,515,518,524,532,534-538,547$. $564,569,576,593$

Hemelingen 606

Heppenheim 343, 397f., 401 f., 443

The Hermitage 151

Hersbruck 163, 165, 285, 290, 293, 313

Hessen 10, 44, 73, 75, 86, 146, 161, 193, 207 . $209,211,216,245,256,317-453,457,459$, $489,510,524,533$

- RB Darmstadt 324f., 335, 340ff., 345, $347 \mathrm{f} ., 353,410,436,442$

- RB Kassel 324f., 327, 335, 341 ff., 345ff., $353,374,410,442$

- RB Wiesbaden 323ff., 327, 335, 341 ff., $346 \mathrm{f} ., 353,403,407,410,432,441 \mathrm{f}$.

Hessisch Lichtenau 34

Hilpoltstein $163,166,285,290,293,313$

Hockenheim 460,510,517

Höchstadt a.d. Aisch 163, 166, 285, 290, 293. 313

Hof $146,163,166,249,285,290,293,308,313$

Hofgeismar 344f., 347f., 397f., 400ff., 444, 447

Hotheim a. Taunus $325,344,346 f$., 396, 398, $401 \mathrm{f} ., 444$

Hotheim i. UFr. 163, 165, 284, 289, 293

Hoya 607

Hünfeld $327,343-347,397 \mathrm{f}$., $400 \mathrm{ff}$., 444, 447

Idstein $325,344,444$

Illertissen $164,168,287,292,295$

Immenstadt i. Allgäu 164

Ingolstadt 146f., 164, 167, 246, 286, 291, 294, 308,314

Inn 146

Inzell 275

Isar 146 
Jena 10

Jouey en Josas 676

Joudreville 321,341

Kaiserslautern 510

Karlsnhe (siehe auch Württemberg-Baden Nord-Baden/LKB Karlsruhe) 63, 70, 107 , 458f., 462, 467 ff., 475, 481 ff., $486 f$., 495 , $500 \mathrm{f}$., $503,505,510,513,515 \mathrm{ff} ., 524,532$, $534-538,541 \mathrm{ff} ., 546,553,560-564,569$. 572 f., $575,577,594$

Karlstadt $163,165,284,290,293,313$

Kassel (siehe auch Hessen/RB Kassel) 324, 340-343, 345 ff., 377-380, 386, 392, $397 \mathrm{f}$., $400 \mathrm{ff} ., 405,411,414,424,436,442,444 \mathrm{f}$. 447,602

Kastel siehe Wiesbaden-Kastel/Mainz-Kastel Kaufbeuren 164, 168, 197, 287, 292, 295, 315

Kelheim 164, 167, 285, 291, 294, 313

Kemnath 164, 166, 291, 294, 314

Kempten 164, 168, 287, 292, 295, 308, 315

Kiel 127

Kirchheim u. Teck 518

Kitzingen $163,165,284,290,293,313$

Knielingen 678

Koblenz 321

Königsberg 4

Königshofen i. Grabfeld 163, 165, 284, 289. 293

Kötzting $164,167,286,291,294,314$

Konstanz 322,572

Korbach 343, 345, 347, 397 f., 402, 444

Kornwestheim 518

Kronach $163,166,290,293,313$

Krumbach (Schwaben) $147,164,168,287$, 292,295

Künzelsau $481 \mathrm{ff} ., 486 f ., 496,500,503,505$. $515,518,535,538,547,569,593$

Kufstein 572

Kulmbach 163, 166, 285, 290, 293, 313

Kurhessen siehe Hessen/RB Kassel

Landau a.d. Isar $146,164,167,285,291,294$, 308,314

Landsberg a. Lech $164,167,239 f ., 246,286$, $292,295,308,314$

Landshut $146,150,164,166,285,288,291$, 294,314

Langenau 518, 593

Lauf a.d. Pegnitz 163, 166, 285, 290, 293, 313

Laufen $164,167,286,292,294$

Lauterbach $341,343,345,347 \mathrm{f}$., $397 \mathrm{f}$., $400 \mathrm{ff}$., 444, 447

Leonberg $481 \mathrm{ff} ., 486 \mathrm{f} ., 495,500 \mathrm{f}$., 503,505 , $515,518,535,538,547,592$

Leoni 244
Les Mesnuls 242

Lesum 609

Lichtenfels $163,166,285,288,290,293,313$

Limburg a.d. Lahn $325,344,346 f ., 388,396$. 398-402, 444

Lindau (Bodensee) 10

Lintfort 341

Linz $146 f$.

Lohr a. Main $163,165,284,290,293$

London 8, 11, 601, 673

Loxstedt $605,609,635,655$

Ludwigsburg 467, $481 \mathrm{ff}$, $486 \mathrm{f}$., 495, $500 \mathrm{f}$., $503,505,515,518,547,556,592$

Ludwigshafen a. Rhein 575

Luxemburg 600

Mainburg 164, 167, 286, 291, 294, 314

Mainfranken siehe Bayem/Unterfranken

Maintaunus 322, 325, 344, 346f., 396, 398402

Mainz 321, 450

- Mainz-Kastel 424

Mallersdorf $164,167,286,291,293,314$

Manchester 148, 457, 510

Mannheim (siehe auch Württemberg-Baden/ Nord-Baden/LKB Mannheim) 135, $321 \mathrm{f}$., $467 \mathrm{ff}$., $475 f$., 481 ff., 486 f., 495, 500 f., 503 , $505,510,515,517,524,532,534-538,541 \mathrm{ff}$. $546,553,556 f$., $560,564,566,572 \mathrm{f}$., 575 . $577 \mathrm{f} ., 595$

- Mannheim-Feudenheim 471, 517, 557, 595

Marburg 324, 327, 340, 342f., 345 ff., $377-$ $380,391,397$ f., $400 \mathrm{ff}$., 405, 414, 439, 442. $444 \mathrm{f} ., 447 \mathrm{f}$.

Marktheidenfeld 163, 165, 284, 290, 293

Markt Oberdorf 164, 168, 287, 292, 295, 315

Martigny les Bains 457

Maxeville 321

Mellrichstadt $163,165,284,289,293$

Melsungen 327, 343 ff., $397 \mathrm{f} ., 400 \mathrm{ff} ., 445,447$

Memmingen 164, 168, 287, 292, 295, 315

Miesbach $162,164,167,275,286,292,294$, 308

Miltenberg $163,165,284,290,293,313$

Mindelheim 164, 168, 287, 292, 295, 308, 315

Minden 52, 56, 61, 104

Mittelfranken siehe Bayern/Mittel- und Oberfranken

Mittenwald 164

Montbard 458

Moosburg a.d. Isar 164

Mosbach $481 \mathrm{ff} ., 486 \mathrm{f} ., 495,500,503,505$, $515,517,547,569,595$

Mühlacker 535, 592

Mühldorf a. Inn $164,167,286,292,294,308$, 314 
Münchberg $163,166,240,285,290,293,313$

München 76,116,127, 138f., 145f., 148, 150153, 156f., 161-164, 167 ff., 171, 174, 179 . 194 ff., $198 f$., 201 ff., 212, 216-220, 222, 226, $230 f$. . 233, 236, 239, 242-249, 251, 255, 260 , $263,269,272,275,278,280 f$., $283 f$., 286289, $291 \mathrm{f}$., 294f., 306, 308, 312, 314

Münsingen 569

Mulde 146

Murnau 164

Nabburg $164,166,286,291,294,314$

Naila $163,285,290,293,313$

Nancy 422

Nassau siehe Hessen/RB Wiesbaden

Neiße 4

Neuburg a.d. Donau 147, 164, 168, 287, 292, 295,315

Neumarkt i. d. OPf. $164,166,286,291,294,314$

Neunburg vorm Wald 164, 166, 285, 291, 294. 314

Neustadt a.d. Aisch $163,166,285,293,313$

Neustadt a.d. Waldnaab $164,166,285,294$, 314

Neustadt a.d. WeinstraBe $10,321,342,427$, 435

Neustadt b. Marburg 429

Neu-Ulm 168, 287, 292, 295, 315

Niederlande $600 f$.

Niederbayern siehe Bayern/Niederbayern und Oberpfalz

Niedersachsen $139,608,620 f ., 623$

Nördlingen $147,164,168,287,294,572$

Nordenham 605, 609, 635

Nordrhein-Westfalen 621

Nümberg 49, 57, 61, 69f., 76, 79, 93, $118 \mathrm{ff}$. $135,137,142,145,163,165,174,196,199$. $201,217,224,232,236,241,243-247,249$, $269,272,278,280,285,287 \mathrm{f}$., 290, $292 \mathrm{f}$., $295,299,306,308,313$

Nürtingen $481 \mathrm{ff}$., $486 \mathrm{f}$., 495, $500 \mathrm{f}$., 503, 505 , $515,518,535,538,547,592$

Oberbayem siehe Bayern/Oberbayern

Oberfranken siehe Bayern/Mittel- und Oberfranken

Oberlahn 325, 344, 346f., 396, 398f., 401 f.

Obernburg a. Main 163, 165, 284, 290, 293

Obernzell 286, 291

Oberpfalz siehe Bayem/Niederbayern und Oberpfalz

Ober-Ramstadt $325,343,445$

Oberreifenberg 369

Obersalzberg 146

Obertaunus $322,325,344,346 f$., 396, 398, $400 \mathrm{ff}$.
Oberursel (Taunus) 353

Oberviechtach $164,166,286,291.294,314$

Obrigheim 556

Ochsenfurt $150,163,165,168,284,290,293$

Oder 4

Öhringen $481 \mathrm{ff} ., 486 \mathrm{f} ., 496,500,503,505$, $515,518,547,569,594$

Österreich $139,146 f ., 162,369,569,577,600$

Offenbach a. Main $322,343,345,347 f$., 363 , $397-402,409,424,445,447$

Oldenburg $605,610,615,617,621 \mathrm{f}$.

Osterhofen 164

Osterholz 603, 605, 608f.

Osterholz-Scharmbeck $604 \mathrm{f} ., 609 \mathrm{f} ., 615$, $630,635,655$

Ostpreußen 4

Paris 8, 458

Parsberg 164, 166, 285, 291, 294, 314

Partenkirchen siehe Garmisch-Partenkirchen

Passau 122, 146f., 164, 166, 249, 285, 288, $291,294,308,314$

Pegnitz 163, 166, 285, 290, 293, 313

Pestinghausen 608

Pfaffenhofen a.d. Ilm 164, 167, 286, 291, $294,308,314$

Pfalz 9f., 319, 321, 432, 585

Pfarrkirchen 164, 167, 285, 291, 294, 314

Pforzheim 481 ff., 486f., 495, 500f., 503, 505, 516f., 546, 573, $594 f$.

Pilsen 148

Plattling 164

Plauen 146

Pommem 4

Potsdam 680

Regen (FluB) 146

Regen $167,286,291,294,314$

Regensburg 146f., 150, 156, 163f., 166, 168, $174,196,198,202,212,217,236,243 f$., 246, $249,256,284$ f., 287 f., 290f., 293, 295, 306, 313

Rehau 163, 166, 285, 290, 293, 313

Reims 15

Reisbach 150

Remagen 10

Rhein $145 \mathrm{f}$.

Rheingau 344,346f., 396, 398-401, 403,450

Rheinhessen 10, 321

Rheinland 5,9,457, 575

Riedenburg 164, 166, 286, 291, 294, 314

Rochefort-en-Yvelines 148, 319f., 341, 360, 438, 457, 460, 510, 559

Roding 164, 166, 286, 291, 294, 314

Romilly-sur-Seine 488,608

Rosenberg (Sulzbach-Rosenberg) 148 
Rosenheim 164, 167, 286, 292, 294, 314

Rotenburg a.d. Fulda 327, 343f., 346, 397 f., $400 \mathrm{ff} ., 445,447$

Rothenburg o. d. Tauber $163,166,285$, 293f., 313

Rottenburg a.d. Laaber $164,167,286,291$. 314

Rottenburg a. Neckar 564

Rüdesheim a. Rhein 325, 344, 346f., 396, $398,401,403,445$

Ruhrgebiet $132,135,137,353,403,602$

Rumelange 341

Saarbrücken 321,343

Saarland 10, 132, 135f., 319, 321, 353, 432. 585,602

Sachsen 675,695

Salzburg 145, 147, 572

Scheinfeld $163,166,285,290,293,313$

Schlesien 4

Schlüchtern $325,344,346 f$., $397 \mathrm{ff}$., 402, 445

Schongau $164,167,286,291,294,308,314$

Schorndorf 517f., 575, 592

Schrobenhausen $147,164,167.286,291,294$. 314

Schwabach 163, 166, 285, 290, 293, 313

Schwaben siehe Bayern/Schwaben

Schwabmünchen $164,168,287,292,295,315$

Schwäbisch Gmund $460,463,467,481 \mathrm{ff}$., 486f., 496, 500, 503, 505, 510, 515, $518 \mathrm{f}$., $534 \mathrm{f}$., 537 f., $542,547,558 \mathrm{f}$., $569,576 \mathrm{f}$., 593

Schwäbisch Hall $\mathbf{4 6 0}, \mathbf{4 6 7}, \mathbf{4 6 9}, \mathbf{4 7 6}, \mathbf{4 8 1} \mathrm{ff}$., $486 \mathrm{f} ., 496,500 \mathrm{f} ., 503,505,510,515,518$, $524,535,538,547,569,578,593$

Schwarzenborn 428

Schweinfurt $145,163,165,275,284,288 f$. 293, 308, 313

Schwetzingen 517,578

Shrivenham $7,148,457$

Sigmaringen 510

Sinsheim 481 ff., 486f., 495, 500, 503, 505, $515,517,547,569,595 \mathrm{f}$.

Sizilien 148

Sonthofen 164, 168, 287, 292, 295, 315

Sowjetische Besatzungszone $4,10,25,131$, $135 \mathrm{ff} ., 141,147,420,424,601,673,711 \mathrm{f}$., $720 f ., 737$

Sprendlingen 325,343

St. Avold 341

St. Quirin 147

St. Savine 608

Stade $605,610,615,617$

Stadtsteinach $163,166,285,290,293,313$

Staffelstein $285,290,293$

Starnberg $164,167,286,291,294,314$
Straubing 147, 164, 167 f.. 240 f., 285. 288. 291, 294, 314

Stuttgart $33,43,48 f ., 52,56,62,71 f ., 99$. 122f., 127, 186f., 206, 208f., 225, 234, 251. $458 f . .461 \mathrm{ff} ., 467 \mathrm{ff} ., 471,474,476 \mathrm{f} ., 481 \mathrm{ff}$. 486f., $495 \mathrm{f} . .499,501,503,505,510 \mathrm{f} ., 514 \mathrm{f}$. 518f., 524, 530, 532-538, 542f., 546, 548 . 552f., 555f., 558-563, 566, 576 ff., 580, 584. $586,591,620,622 \mathrm{f}$.

Sulzbach (Sulzbach-Rosenberg) 291, 345

Sulzbach-Rosenberg 164, 166, 286, 294. 314

Syke 608

Tauberbischofsheim 482f., 486f., 495, 500. 503, 505. 515, 517. 535, 538, 547, 569, 577. 595

Taunus siehe Maintaunus, Obertaunus. Untertaunus

Thüringen $10,139,146,593,675,695$

Tirlemont 341

Tirschenreuth $164,166,285,291.294,314$

Töging a. Inn 276

Traunstein $164,167,286,292,294,314$

Trier 151,321

Trostberg 164, 308

Troyes 148,458

Tschechoslowakei $147 \mathrm{f} ., 179,695$

Tübingen 521, 576

Uffenheim 166, 290, 293

Ulm $145,462,467 \mathrm{ff} ., 475,481$ ff., $486 f$., 496 , $500 f ., 505,515,518,524,532,534-538,543$, $547,560,564,569,576,593$

Unterfranken siehe Bayern/Unterfranken Untertaunus $325,344,346 f$., 397-400, $402 \mathrm{f}$.

Usingen $325,344,346 f ., 397-400,402,445$

Uttenreuth 168

Vaihingen a.d. Enz 481 ff., $486 f$., 495, 500, $503,505,515,518,535,538,547,592$

Vegesack 609,612

Verden (Aller) 607

Verdun 341

Versailles 14, 676,678

Viechtach $167,286,291,294,314$

Vilsbiburg $164,291,294,314$

Vilshofen $164,167,286,291,294$

Vohenstrau B 164, 166, 286, 291, 294, 314

Volkmarsen 424

Waiblingen $481 \mathrm{ff}$., 486f., 495, 500, 503, 505 , $515,518,547,569,592$

Waldeck $343 \mathrm{ff}$., 347, $397 \mathrm{f}$., $400 \mathrm{ff}$., 447

Waldmünchen $164,166,286,291,294,314$

Wasserburg a. Inn 150, 164, 167f., 286, 292 , $294,308,314$ 
Wegscheid 164, 167, 286, 291, 294, 314

Weiden 164, 166, 275, 285, 291, 294, 314

Weihenstephan 306

Weilburg 325, 344, 346f.. 396, 398, $401 \mathrm{f}$. . $444 f$.

Weilheim i. OB $164,167,286,288,291,294$, 308

Weimar 10,139

Weinheim 517,577

WeiBenburg i. Bayern $163,166,285,290$, 293, 313

Weißenhorn 164

Wertheim 517,577

Wertingen 147, 164, 168, 287, 292, 295

Wesermarsch 603f., 608ff., 615,630

Wesermünde siehe Bremerhaven

Wetzlar 325, 343, 346f., 353, 396, 398f., $401 \mathrm{f} ., 445$

Wiesbaden (siehe auch Hessen/RB Wiesbaden) $54,63,70 \mathrm{f}$., 107f., 127, 319, $321 \mathrm{f}$., 325 ff., 340, 342f., 345 ff., 361, 368, 374, 376$380,386,391 \mathrm{f} ., 396-400,402,405,409,414$, $420,431,436,439,442,445,447,511,555$

- Wiesbaden-Kastel 424

Wiesloch 517

Windsheim $163,285,293$

Winterscheid 9

Witzenhausen 327, 343f., 346, $397 \mathrm{f}$., $400 \mathrm{ff}$., 445, 447

Wolfhagen 325, 343f., 346f., $397 \mathrm{f}$, $400 \mathrm{ff}$., 445, 447

Wolfratshausen 148, 164, 167, 286, 292, 294. 314
Wolfstein $167,286,291,294,314$

Worms 145

Württemberg $321,457 \mathrm{f} ., 460,475,496,509 \mathrm{f}$., $520,536,541,562,585,588$

Württemberg-Baden $10,44,73,75.87,123$. $161,193,207,209,211,216,245,256,368 \mathrm{f}$. $374,376,384,455-596$

- Nord-Baden 459, 466, 469 f., 477. 512 f., 516 f., $520,524,526,528 f$., 532 . 541 f., 553, 560 ff., 569, 571-577, 585, 594 . $5 \%$

- LKB Karlsruhe 461, 466, 495, $510 f ., 519 \mathrm{f}$., $522,528,542,563,572 \mathrm{f}$., 575,594

- LKB Mannheim 461, 466, 495, 510f., $517,519,528,542,562 f$., $569,572 f ., 594$

- Nord-Württemberg $139,459,466,470$, 510 ff., 519, 524, 526, 528, 542, 559, 561, 563 , $569,573,575$ ff., 591-594

Württemberg-Hohenzollern 457, 510,576, 585

Würzburg 145, 153, 156, 162f., 165, 174, 196, $204,217,236,240,243$ f., 249, 256, 284, 288 ff., 293, 295, 306, 308, 312

Wunsiedel $163,166,285,290,293,313$

Zeil 198

Ziegenhain 325, $343 \mathrm{f}$., 346f., $397 \mathrm{f} ., 400 \mathrm{ff}$., $428,445,447$

Ziemetshausen 150,168

Zwiesel 164,286, 294 
